\title{
NASA Thermographic Inspection of Advanced Composite Materials
}

\author{
K. Elliott Cramer* \\ *National Aeronautics and Space Administration, Langley Research Center, \\ Hampton, VA USA
}

\begin{abstract}
As the use of advanced composite materials continues to increase in the aerospace community, the need for a quantitative, rapid, in situ inspection technology has become a critical concern throughout the industry. In many applications it is necessary to monitor changes in these materials over an extended period of time to determine the effects of various load conditions. Additionally, the detection and characterization of defects such as delaminations, is of great concern. This paper will present the application of infrared thermography to characterize various composite materials and show the advantages of different heat source types. Finally, various analysis methodologies used for quantitative material property characterization will be discussed.
\end{abstract}

\section{Introduction}

Infrared thermography has proved to be an effective tool in the inspection of composite materials. By providing either a single-sided or a two-sided inspection, the presence and growth of defects in aerospace composites can be evaluated and used to estimate the remaining life of these materials. Thermography can be performed using a variety of heat sources including flash heating (short pulse), step heating (long pulse) and spatially shaped heat sources, thus providing a inspection tool that is applicable to a wide range of material properties, thicknesses and defect types.

Thermography has many advantages over more traditional inspection methods. For example, ultrasonic (UT) inspection methods typically require the use of a coupling medium (either water or some other fluid), which can present difficulties for some materials and can make in situ inspection significantly more complicated. Further, UT inspections consist of scanning a small diameter transducer over the surface of the structure, this requires expensive, automated scanning equipment and can be quite time consuming. Thermography, on the other hand, can rapidly image large areas of the structure with little or no surface preparation. In a typical inspection it is possible to image a $1 \mathrm{~m}^{2}$ area in approximately 20 seconds.

Radiographic techniques, such as x-ray imaging, are another class of traditional inspection methods used for composite materials. While radiography can be quite effective in characterizing the size and location of defects, safety becomes a driving factor when performing large-scale inspections. Additionally, two-sided access to the structure is almost always required for this technique, making in situ inspection difficult, if not impossible, in many applications. Thermography has the advantage of being able to perform single-sided measurements on many materials and can be carried out without the safety concerns associated with ionizing radiation.

\section{Application of thermography to graphite-epoxy composites}

The implementation of NASA's infrared thermography system use for singlesided data acquisition on graphite-epoxy composite materials is shown in Fig. 1. The IR imager is a commercial radiometer with a cooled $256 \mathrm{H} \times 320 \mathrm{~V}$-element InSb (Indium - Antimonide) focal plane array detector. The radiometer's noise equivalent 
temperature difference (NE $\Delta \mathrm{T})$, cited by the manufacturer, is $0.025^{\circ} \mathrm{C}$ when operating the detector in the 3 to 5 micrometer wavelength range. The radiometer produces images at both 30 frames per second output (video frame rate, in an RS170, format compatible with standard video equipment) and 60 frames per second output in a 12-bit, RS422 digital format. External optics, consisting of a wide-angle lens, using germanium optical elements, were used to increase the system field-ofview by a factor of approximately two. The expanded field-of-view of this lens is $41^{\circ}$ horizontally and $31^{\circ}$ vertically.

Heating of the inspection surface is produced by focusing two 750-W quartz lamps on the inspection area using parabolic reflectors. Spurious reflections were reduced or eliminated by adjusting the camera's incident angle to the surface, filtering the heat source output with Plexiglas ${ }^{\circledR}$ filters and by controlling the reflections from the surrounding environment with gobos made from foam-core poster board.

Quantitative time based analysis requires synchronization between the IR imager and the heat source. This synchronization is achieved by computer control of the application of heat and the data acquisition. All inspection sites were heated for 20 seconds, and thermal data were acquired during both the heat-up and cool-down of the inspection area (40 seconds total for each site). Images were recorded at a frame rate of 15 frames per second. The camera was mounted on a tripod in order to maintain a consistent focal length of $51 \mathrm{~cm}$ to the surface where possible. For all cases presented in this paper, the maximum surface temperature change of the specimen above ambient was less than $10^{\circ} \mathrm{C}$.
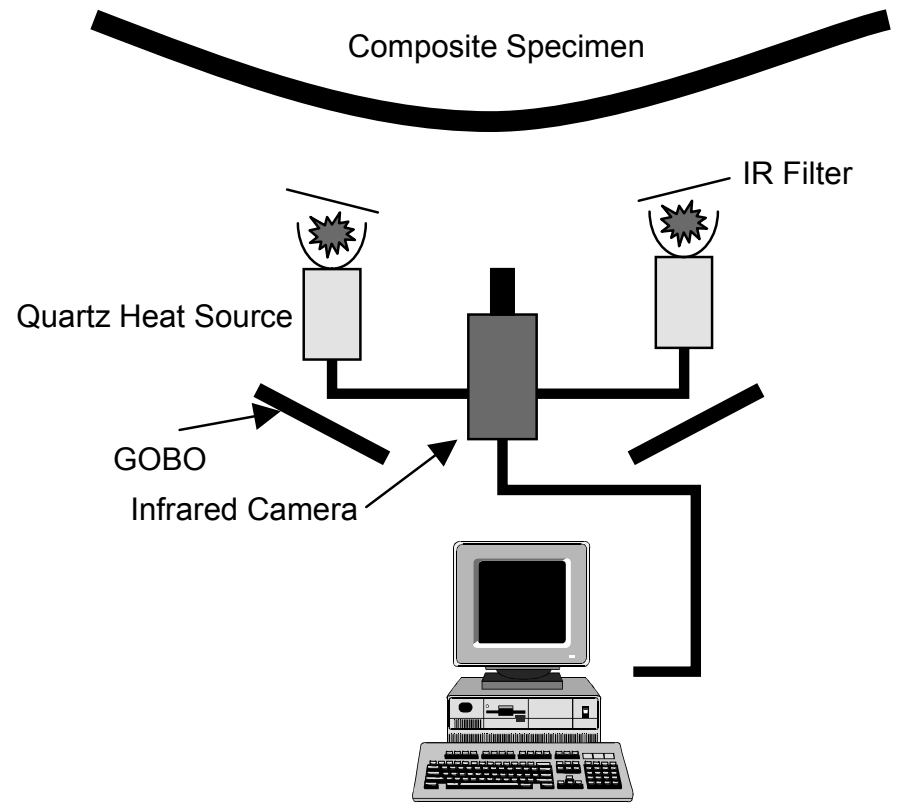

Data Acquisition Computer

Fig. 1 - Drawing of thermal inspection system used for the single-sided inspection of graphite-epoxy composite fuselage.

The time-series of thermal images was processed to produce single images related to the rate of thermal diffusion into the material. Calculation of the 
instanteous time derivative has been shown to be an effective means of analyzing thermal images to locate delaminations [1], [2]. The processing consists of approximating a normalized time derivative 15 seconds after the onset of heating (both the heat-up and cool-down data is used to calculate the time derivative). To implement calculation of the time derivative efficiently, a weighted sum can be formed which yields an approximation to the time derivative. The weights for the sum are defined by the constant $f(i, j, n)$ as follows[3]:

$$
f(i, j, n)=\frac{12 i-6 n-6}{n^{3}-n}-30(2 j+n+1)\left(\frac{6 i^{2}-6 i+n^{2}+3 n-6 i n+2}{n^{5}-5 n^{3}+4 n}\right)
$$

where $i$ is the number of the image for which the weight is being calculated, $j$ is the image number where the time derivative is desired and $n$ is the number of images in the sequence. To help reduce the effects of uneven heat application and any spatial variations in the emissivity of the specimen, the resulting time derivative is normalized using the last image of the time-series. Therefore the final reduced thermal image is determined from the sum:

$$
\frac{\mathrm{d}}{\mathrm{dt}} \mathrm{T}(\mathrm{j}) \approx \sum_{\mathrm{i}=1}^{\mathrm{n}}\left(\frac{\mathrm{f}(\mathrm{i}, \mathrm{j}, \mathrm{n}) \mathrm{T}(\mathrm{i})}{\mathrm{T}(\mathrm{n})}\right)
$$

where $\mathrm{dT}(\mathrm{j}) / \mathrm{dt}$ is the approximate time derivative at image $\mathrm{j}, \mathrm{T}(\mathrm{i})$ is the indexed series of temperature images and $T(n)$ is the last image in the sequence, which is used for normalization.

This methodology was recently applied to the inspection of a composite vehicle under development by NASA. NASA's X-37 is an advanced, reusable launch vehicle, designed to operate in both the orbital and reentry phases of flight. The fuselage of the vehicle is constructed of graphite-epoxy composite materials.

Fig. 2 shows typical results obtained using the infrared technique described in this paper. Separate thermographic inspections were performed on approximately $90 \%$ of the external skin of the X37 both before and after full-scale load testing of the vehicle.

\section{Application of thermography to reinforced carbon-carbon composites}

In applying thermal imaging to reinforced carbon-carbon (RCC) composites the only modification to the system described above was to replace the quartz heating with flash heating. In a typical application, two 6400 Joule xenon photographic flash tubes are used to produce impulse heating in the specimen. Fig. 3 shows the experiment setup used for through-transmission imaging of the RCC panels. The RCC specimen in these tests was a leading edge wing panel removed from the space shuttle for impact testing. The specimen examined was approximately $69 \mathrm{~cm}$ in length and approximately $0.61 \mathrm{~cm}$ in thickness across the upper and lower panel surfaces. Processing of the thermal response of the specimen to impulse heating was performed using two different methods. First, for a rapid, qualitative, characterization of the thermal response, a median difference algorithm was performed on the thermal data. The resulting image is produced by performing a linear least squares curve fit of the thermal response of the specimen with the median thermal response of a reference region (typically a region of known "good" material or a calibration standard). 


\section{http://dx.doi.org/10.21611/qirt.2004.a}

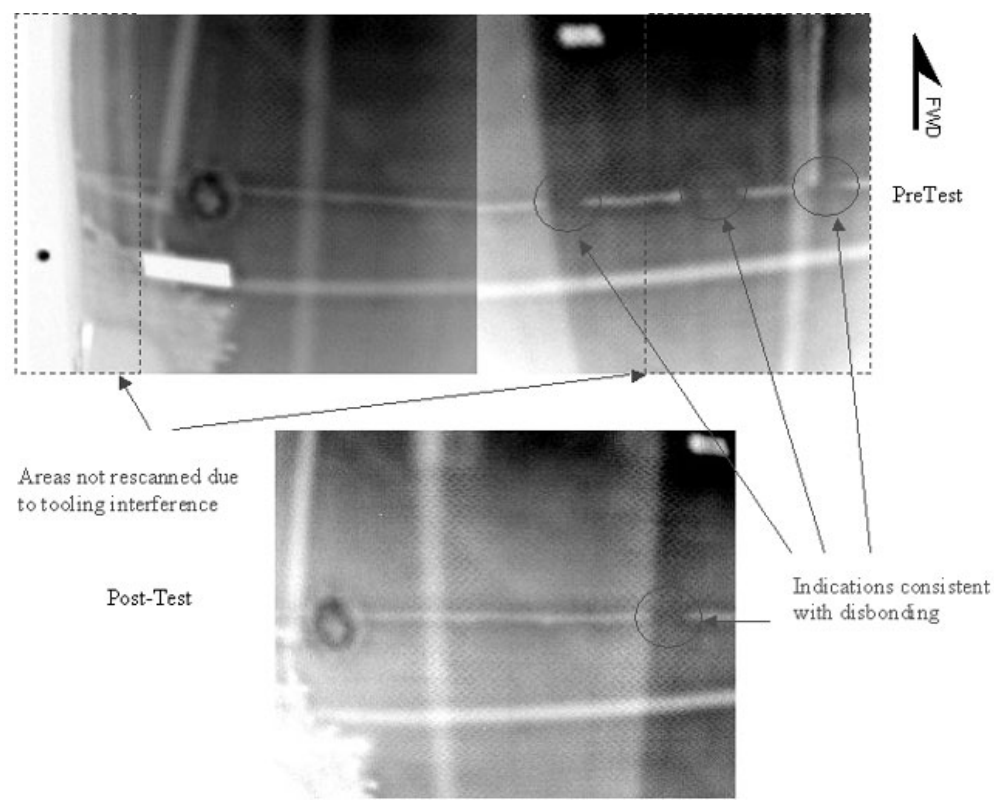

Fig. 2 - Thermal time derivative image of the underside of $X-37$ vehicle, right side of Center-line as viewed facing forward on the vehicle. Comparison of pre- and postload test inspection results showing no change in the indications consistent with disbonding.

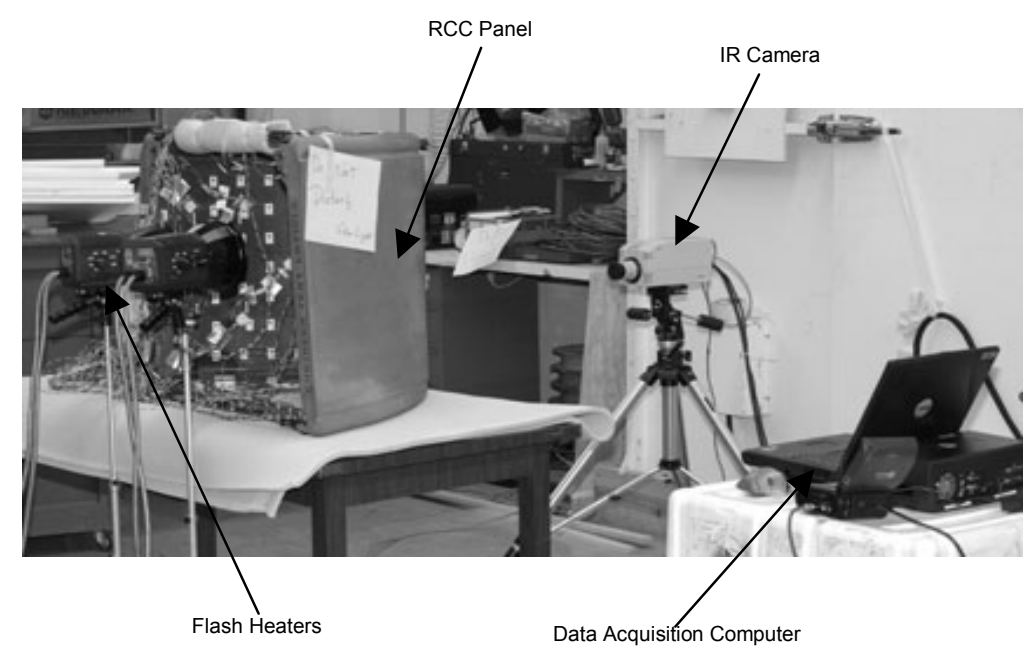

Fig. 3 - Photograph of experimental setup used in the inspection of RCC composite aerospace panels. 


\section{http://dx.doi.org/10.21611/qirt.2004.a}

The median difference image $S(x, y)$ is given by:

$\mathrm{S}(\mathrm{x}, \mathrm{y})=\frac{\sigma_{\mathrm{d}}(\mathrm{x}, \mathrm{y})}{\sigma_{\mathrm{S}}(\mathrm{x}, \mathrm{y})}$,

where $\sigma_{d}$ is the standard deviation of the difference between the curve fit and the actual temperature response and $\sigma_{s}$ is the standard deviation of the original temperature response for each pixel location in the image.

The RCC composite specimen in this inspection was subjected to a high velocity impact. The specimen was impacted with a $5.08 \mathrm{~cm} \times 17.78 \mathrm{~cm} \times 24.38 \mathrm{~cm}$ block of foam weighing 73.6 grams projected at a velocity of $355.7 \mathrm{~m} / \mathrm{sec}$. Fig. 5 shows a typical thermal image of RCC materials processed using the median difference technique. As can be seen in fig. 5 the damage created by the foam impact extends approximately $43.2 \mathrm{~cm}$ from the outboard rib toward the center of the RCC specimen at the apex of the leading edge.

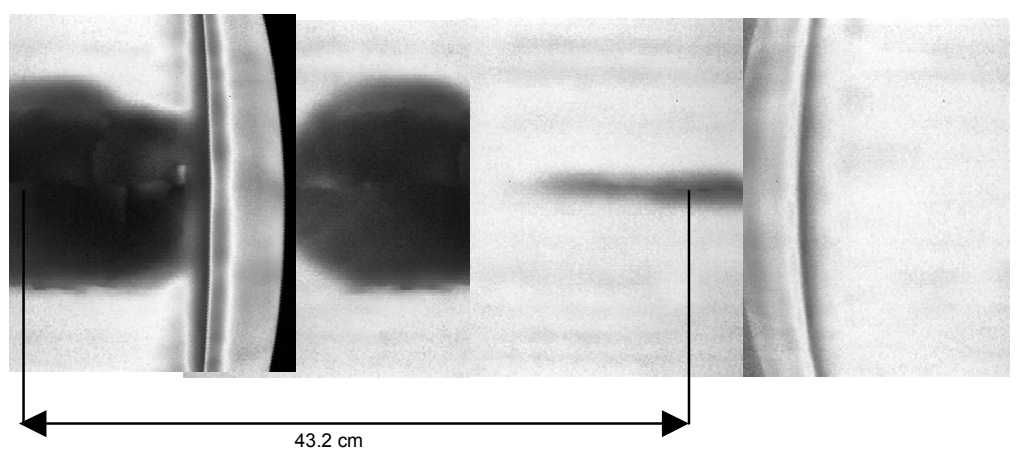

Fig. 5 - Collage of four median difference thermal images of an RCC composite panel showing the delamination (dark region) that resulted from the high velocity foam impact.

The second processing method that was applied is to quantitatively calculate the thermal diffusivity of the specimen of interest. The thermal diffusivity is obtained by performing a nonlinear least squares curve fit of the thermal response of the specimen using the following model [4]:

$\mathrm{T}(\mathrm{L}, \mathrm{t})=\frac{\mathrm{a}}{\sqrt{\mathrm{t}}}\left[\sum_{\mathrm{n}=0}^{\infty} \exp \left(-\frac{(\mathrm{n}+1)^{2} \mathrm{~b}}{\mathrm{t}}\right)\right]$

where, $\mathrm{a}=\frac{2 \mathrm{Q}}{\rho \mathrm{c} \sqrt{\pi \alpha}}$

and, $b=\frac{L^{2}}{4 \alpha}$.

In Eqs (4-6) $\alpha$ is the thermal diffusivity, $\rho$ is the density, c the specific heat, $L$ the specimen thickness, $Q$ the input heat flux and the time. Fig. 6 shows the results of the curve fit to obtain the parameter $b$, which is inversely proportional to the thermal diffusivity of the specimen. The average thermal diffusivities for two regions, shown in fig. 6 , of the specimen were calculated based on Eq. (6). For region \#1 the diffusivity was found to be $0.033 \pm 0.004 \mathrm{~cm}^{2} / \mathrm{s}$ and $0.067 \pm 0.003 \mathrm{~cm}^{2} / \mathrm{s}$ for region \#2. 


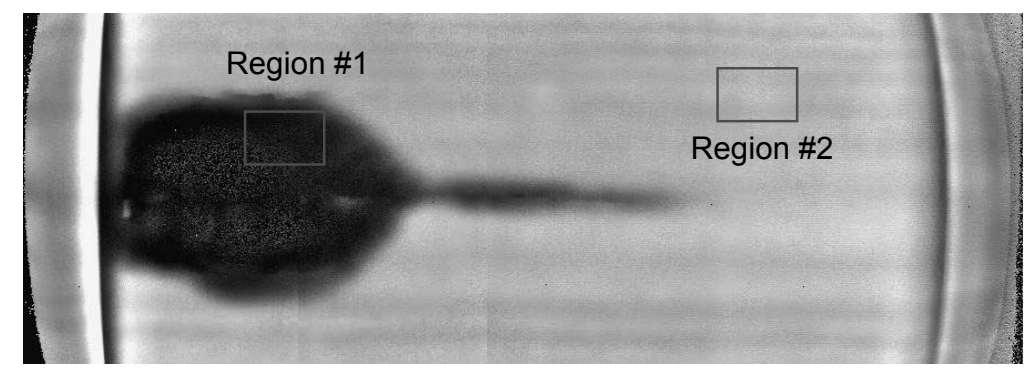

Fig. 6 - Collage of four diffusivity images of an RCC composite panel showing the delamination (lower diffusivity region) that resulted from the high velocity foam impact. Two regions are shown for comparison of the average thermal diffusivity values.

\section{Conclusions}

Infrared thermography has been shown to be effective for the characterization of a wide range of composite materials. For thicker composites where access to only one side of the structure is possible, long pulse thermography and time derivative analysis effectively images disbonding between the composite skin and substructure. In the case of a graphite-epoxy fuselage with disbonding between the composite skin and subsurface structural elements, the disbonds were successfully imaged both before and after full-scale load testing of the vehicle. For thinner composites, or composites where near surface features are of significant interest single sided flash thermography using median difference analysis provides a very rapid and effective inspection method. This method was shown to be capable of mapping the damage created in a RCC wing leading edge specimen subjected to a high velocity impact from a foam projectile. Finally, where complete analysis of the bulk material is required, thermal diffusivity imaging provides that capability and can be used to characterize either disbonding or changes in the material properties quite effectively. For the same RCC wing leading edge specimen a change in thermal diffusivity of $0.034 \mathrm{~cm}^{2} / \mathrm{s}$ was observed between an undamaged and a damaged region of the specimen.

\section{Acknowledgements}

The author would like to acknowledge Mr. Fred Hibbard and Mr. Kenneth L. Hodges, both of Lockheed-Martin Space Operations, for their support of the data acquisition software and field inspections.

\section{REFERENCES}

[1] W.P. Winfree, B.S. Crews, H.I. Syed, P.H. James and K.E. Cramer, Proceedings of the $37^{\text {th }}$ International Instrumentation Symposium, (ISA, Research Triangle Park, 1990), p. 881.

[2] P.A. Howell, W.P. Winfree and B.S. Crews, Review of Progress in Quantitative Nondestructive Evaluation, Vol. 10B, ed. D.O. Thompson and D.E. Chimenti (Plenum, New York, 1991), p. 1367.

[3] K.E. Cramer, P.A. Howell and H.I. Syed, Proceedings SPIE - Thermosense XVII,Vol. 2473, ed. S. A. Semanovich (SPIE, Bellingham, 1995), p. 226.

[4] W.P. Winfree and D.M. Heath, Proceedings SPIE - Thermosense XXIII, Vol. 3361, ed. J. R. Snell, (SPIE, Bellingham, 1998), p. 282. 